\title{
Kerker Effect in Ultrahigh-Field Magnetic Resonance Imaging
}

\author{
Marc Dubois, ${ }^{1}$ Lisa Leroi, ${ }^{2}$ Zo Raolison, ${ }^{2}$ Redha Abdeddaim, ${ }^{1,}$ Tryfon Antonakakis, ${ }^{3}$ Julien de Rosny, ${ }^{4}$ \\ Alexandre Vignaud, ${ }^{2}$ Pierre Sabouroux, ${ }^{1}$ Elodie Georget, ${ }^{2, \dagger}$ Benoit Larrat, ${ }^{2}$ Gérard Tayeb, ${ }^{1}$ Nicolas Bonod, ${ }^{1}$ \\ Alexis Amadon, ${ }^{2}$ Franck Mauconduit, ${ }^{5}$ Cyril Poupon, ${ }^{2}$ Denis Le Bihan, ${ }^{2}$ and Stefan Enoch ${ }^{1, \$}$ \\ ${ }^{1}$ Aix Marseille Univ, CNRS, Centrale Marseille, Institut Fresnel, 13013 Marseille, France \\ ${ }^{2}$ CEA, DRF, JOLIOT, NeuroSpin, UNIRS, Université Paris-Saclay, 91191 Gif-sur-Yvette Cedex, France \\ ${ }^{3}$ Multiwave Innovation AG, 1228 Geneva, Switzerland \\ ${ }^{4}$ ESPCI Paris, PSL Research University, CNRS, Institut Langevin, 75005 Paris, France \\ ${ }^{5}$ Siemens Healthineers, 93210 Saint Denis, France
}

(Received 27 April 2018; revised manuscript received 26 July 2018; published 27 September 2018)

\begin{abstract}
Ultrahigh-field (UHF) magnetic resonance imaging (MRI) systems are getting a lot of attention as they ensure high intrinsic signal-to-noise ratio resulting in higher spatial and temporal resolutions as well as better contrast. This promises improved clinical results with regard to morphological as well as functional and metabolic capabilities. Traditionally, MRI relies on volume coils (birdcage) able to deliver a homogeneous radio frequency field exciting the nuclei magnetic spin. However, this strategy is hindered at UHF because of the rf field inhomogeneities yielded by the increased Larmor frequency. A standard approach consists of inserting passive dielectric elements within the volume coil in order to locally enhance the rf field and mitigate these inhomogeneities. However, the lack of control over their electromagnetic properties prevents the development of optimal solutions. Here, a single meta-atom is used to achieve efficient and tunable rf field control in UHF MRI. We demonstrate theoretically and experimentally a full overlap between the electric dipolar and magnetic dipolar resonances of the meta-atom. This interaction is precisely tuned to reach the so-called Kerker scattering conditions when illuminated in the near field by a birdcage coil. At these conditions, a strong enhancement or suppression of the rf field is achieved in the vicinity of the meta-atom within the MRI volume coil.
\end{abstract}

DOI: 10.1103/PhysRevX.8.031083

Subject Areas: Metamaterials

\section{INTRODUCTION}

Since its discovery in the early 1970s, magnetic resonance imaging (MRI) scanners have become one of the most efficient diagnostic tools available for medical imaging. Also, over time, their magnetic field strength $\left(B_{0}\right)$ has been steadily increased to enhance signal-to-noise ratio (SNR) [1]. Such a gain could be used to improve image sensitivity and spatial and/or temporal resolutions. Ultrahigh-field (UHF) MRI $\left(B_{0} \geq 7 \mathrm{~T}\right)$ is identified as a potential candidate to detect early symptoms of serious neurodegenerative pathologies such as Alzheimer's disease [2]. This increasing $B_{0}$ induces a proportional increase of the Larmor or precessional frequency of the nucleus spin

\footnotetext{
*redha.abdeddaim@fresnel.fr

${ }^{\dagger}$ Now at: Multiwave Innovation, Marseille, France.

†stefan.enoch@fresnel.fr
}

Published by the American Physical Society under the terms of the Creative Commons Attribution 4.0 International license. Further distribution of this work must maintain attribution to the author(s) and the published article's title, journal citation, and DOI. and a decrease of the corresponding wavelength $\left(\lambda_{0}\right)$ for the radio-frequency excitation field. Consequently, the typical human body size (diameter of the head is around $16 \mathrm{~cm}$ ) becomes non-negligible compared to the wavelength $(\lambda$ at $7 \mathrm{~T}$ is equal to $11 \mathrm{~cm}$ in the body) and interferences can occur leading to bright and dark zones. At $7 \mathrm{~T}$, rf field distribution in the human head presents a bright center and a weak periphery when transmit volume coils are used [Fig. 1(a)] [3]. This induces locally major losses in contrast or shadowing on the images depending on the MR acquisition strategy [white arrows in Figs. 1(b) and 1(c)]. Note that these losses appear in regions where the rf field drops below a threshold value around $40 \%$ of the maximum value. Such artifacts make UHF MRIs inadequate for several diagnostic purposes and represent a major bottleneck for a global application to the clinical environment.

Different approaches have been proposed to tackle this problem. The first one consists of using high dielectric constant pads surrounding the region of interest to locally enhance the rf field $[4,5]$. Unfortunately, major drawbacks prevent their use in high-field clinical routine: performance decay over time and ecotoxicity of the active substances used conventionally [6]. 

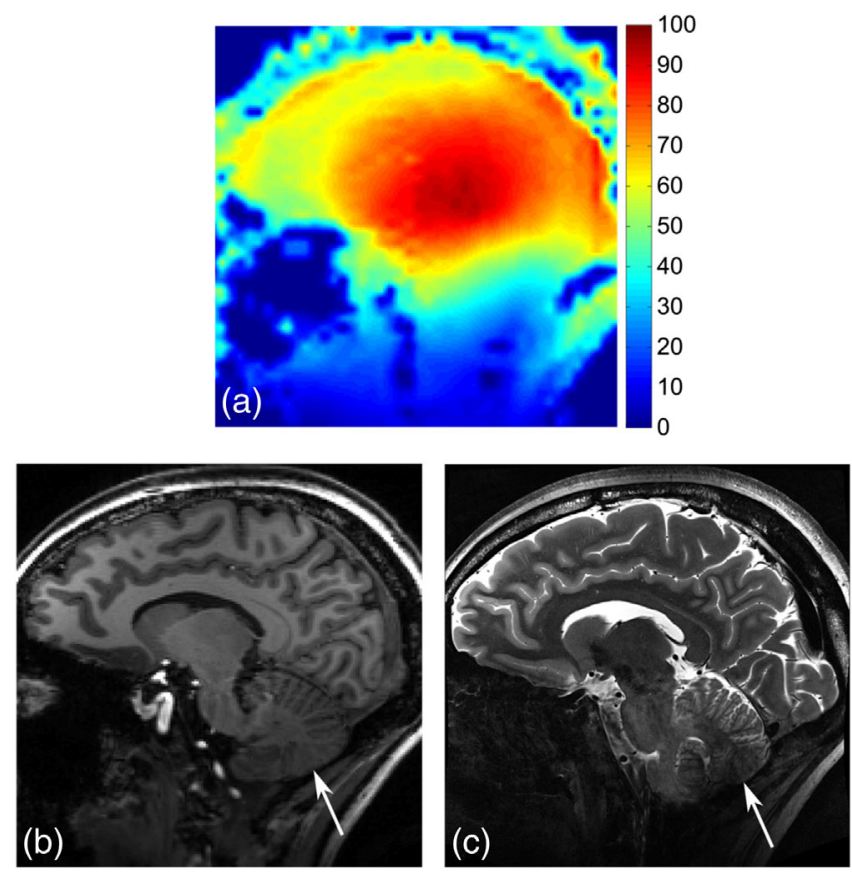

FIG. 1. $7 \mathrm{~T}$ sagittal MR images of a human brain slice obtained with various acquisition sequences: (a) Flip-angle (FA $\propto B_{1}^{+}$) map acquired using the actual flip-angle imaging sequence (color bar in degree). (b) T1-weighted magnetization-prepared rapid gradient echo sequence. (c) T2-weighted turbo spin echo sequence. Those images have been acquired with a birdcage coil for excitation and a 32-loops phased array for reception. Notice (a) the large $B_{1}^{+}$field heterogeneity over the head (dispersion of $\sim 30 \%$ ). When FA is too far from the target, as is the case here in the cerebellum (white arrows), it leads to loss in contrast (b) or even worse to shadowing (c) depending on the acquisition strategy.

Another solution, called parallel rf transmission, has been introduced. It consists in placing several independent rf transmitters around the subject, instead of a single one on standard MR systems. Taking advantage of rf interference and dynamic modulation during the excitation, it is possible to obtain a much more homogeneous excitation [7]. These additional degrees of freedom provide a better control of the magnetization but also raise challenging problems in terms of workflow and safety. None of the solutions described above has been fully satisfactory for UHF MRI. To date, only the single transmit channel volume coil has received approval for 7 T MRI clinical applications [8].

Recently, metamaterials have been introduced to control rf fields in the MRI context. Metamaterials are composite materials, most often made of resonant cells, whose effective properties mimic a homogeneous material that is not available in nature. Several implementations of metamaterials have been used to homogenize the field, enhance the SNR and the penetration depth for moderate field MRI [9-16] and to a lesser extent for UHF MRI to enhance performances and the decoupling of surface coils [13,17-20]. However, none of these works has tackled the problem of the field inhomogeneity in UHF MRI volume coils. The latest developments propose to merge a metamaterials approach and high permittivity dielectric materials in order to access multiple degrees of freedom for the local enhancement of the rf field in MRI volume coils [21].

A crucial aspect for the control of radiation lies in the ability to tailor simultaneously the electric and magnetic responses of a single scatterer. This was first theoretically envisioned by Kerker et al. [22]: for a particle with specific values of permittivity and permeability, the fields scattered by the induced electric and magnetic dipoles can interfere, thus leading to strong scattering anisotropy. This scattering anisotropy was recently measured in the microwave regime [23] and is referred to as first and second Kerker conditions corresponding to a zero backward scattering and a nearzero forward scattering depending on the excitation frequency. The Kerker conditions can also be extended to the case of a near-field excitation when considering an electric dipole emitter coupled with a scatterer with strong electric and magnetic responses [24]. We want to take advantage of this physical effect in the rf range in order to control the rf field distribution in a MRI volume coil. However, the large dielectric constant required and the lack of space between the coil and the subject led us to dismiss the use of dielectric materials.

In this paper, we propose a new passive method based on metamaterials to improve the rf field control in UHF volume coils. We demonstrate that the rf field distribution of a birdcage coil can be controlled by inserting a metaatom between the sample and the coil. A meta-atom can be designed using a single resonator element to build up a more complex unit cell using a coupling mechanism [25]. The meta-atom considered is based on a set of four hybridized resonant metallic wires. It is referred to as a hybridized meta-atom (HMA) in the following. First, we use an analytic approach based on impedance matrices in order to characterize the hybridization mechanism and derive the Kerker conditions. This model is used to describe the interaction between the HMA and a plane wave excitation as well as a near-field excitation (i.e., birdcage coil). Finally, we demonstrate experimentally that the HMA can controllably redistribute and reshape the rf electromagnetic field within a commercial birdcage coil in a $7 \mathrm{~T}$ MRI scanner.

\section{RESULTS}

\section{A. Kerker conditions from HMA with far-field illumination}

A structure composed of several coupled resonators will present a new set of hybrid resonances. This effect is particularly striking when strong near-field coupling occurs as the distance between resonators decreases with respect to the wavelength considered. We apply this strategy in order to design our HMA structure with strong electric and 
magnetic effective induced dipoles. It is composed of four $z$-oriented thin metallic wires located at the corners of a rectangle of sides $d_{1}=1.5 \mathrm{~cm}$ and $d_{2}=2 \mathrm{~cm}$. A single wire mainly interacts with the electric field along its axis and presents an electric resonance when its length approaches a multiple of half a wavelength. However, when several wires are used with subwavelength separation, the coupling or hybridization between the wires cannot be neglected when describing the response of the whole structure. Scattering and interactions of electromagnetic fields with objects are often characterized by the electric and magnetic polarizabilities. Although it is useful while studying scattering from Mie particles [26], the impedance matrix approach borrowed from the antenna community is well suited for our problem. This approach is based on the derivation of the impedance matrix $Z_{i j}$ of the system, where $i$ and $j$ stand for the resonators' indexes. This matrix connects the different currents and voltages present in every element of the array and can be used to predict the behavior of complex arrangements of resonators [27]. The diagonal elements of this matrix give the selfimpedance of each isolated wire whereas the off-diagonal elements are the mutual impedances between the wires. This mutual impedance represents the open circuit voltage induced on a wire if one of its neighbors is excited. Thus, the contribution of the near field to the mutual impedance directly depends on the separation distance between the wires considered. As we consider only wires of length shorter than a full wavelength (almost $1 \mathrm{~m}$ at $297.2 \mathrm{MHz}$ ), we can assume that the current along $z$ is a sinusoidal function as in Eq. (1):

$$
I(z)=I_{0} \frac{\sin \left[k\left(\frac{L}{2}-|z|\right)\right]}{\sin \left(k \frac{L}{2}\right)} .
$$

Within this assumption, one can calculate the self- and mutual impedances of the system from a standard analytical derivation described in Eq. (2), where $E_{i j}$ corresponds to the $z$ component of the electric field produced by the wire $j$ along the wire $i$ :

$$
Z_{i j}=-\frac{1}{I_{0 i} I_{0 j}} \int_{-L / 2}^{L / 2} E_{i j}(z) I_{i}(z) d z
$$

The analytical derivation of Eq. (2) is described in full detail in the Supplemental Material [28]. Once all the $Z$-matrix elements are evaluated, it is possible to access the electrical currents on every wire for a given driving voltage (currents and voltages are vectors) with the relation $I=Z^{-1} V$. As we would like to characterize the response of passive wires (i.e., short-cut antenna), it is necessary to introduce a fifth wire represented in green in the sketch in Fig. 2(a) as a driven element while the HMA wires remain passive. In this specific case, the $Z$ matrix is a $5 \times 5$ matrix that can be computed at a frequency of $297.2 \mathrm{MHz}$ (Larmor frequency of the hydrogen nucleus at $7 \mathrm{~T}$ ). When placed in the far field of the HMA, the driven element acts as a plane wave source. We can access the current amplitude and phase in each wire of the HMA by inverting the $Z$ matrix and use $V=[1 ; 0 ; 0 ; 0 ; 0]$ as a voltage input (indices from 2 to 5 represent the short-cut HMA wires). A final step consists of calculating the radiated power $P_{\text {rad }}$ from the HMA. This requires the complex current and the positions of the HMA wires yet again assuming sinusoidal current distribution along $z$. We first calculate the radiation intensity $U(\theta, \phi)$ from the four HMA wires and then perform the integration over all solid angles to obtain the total radiated power $P_{\text {rad }}$ :

$$
\begin{aligned}
U(\theta, \phi)= & \frac{1}{8 \pi^{2} c \epsilon_{0}} \mid \sum_{p=2}^{N} I_{p} \frac{\cos \left(k \frac{L}{2} \cos \theta\right)-\cos \left(k \frac{L}{2}\right)}{\sin \left(k \frac{L}{2}\right) \sin \theta} \\
& \times\left. e^{j k \sin \theta\left(x_{p} \cos \phi+y_{p} \sin \phi\right)}\right|^{2}, \\
& P_{\mathrm{rad}}=\int_{0}^{\pi} \int_{0}^{2 \pi} U(\theta, \phi) \sin \theta d \theta d \phi .
\end{aligned}
$$

As shown in Fig. 2(b), we calculate the power radiated by the HMA (solid red line) as a function of the wire length for a given plane wave excitation. A control case is done with a single wire located at the origin. One can observe that the HMA response shows two maxima, each one associated with a different hybrid resonance. The first broad peak at $0.47 \mathrm{~m}$ corresponds to the excitation of a dipolar electric mode where the four wires are excited in phase with the same amplitude (i.e., symmetric mode). The second sharp peak at $0.5 \mathrm{~m}$ corresponds to a dipolar magneticlike mode where the currents between the wires in $y=0$ and $y=d_{2}$ are out of phase (i.e., antisymmetric mode). Because of the strong coupling within the HMA, the magnetic dipolar resonance presents a very high quality factor. On the other hand, the electric dipolar resonance of the HMA is significantly wider than the one obtained from a single wire. The structure of these hybrid resonances has been described previously for single magnetic mode volume coil applied to small animal MRI [17].

Interactions of electric and magnetic modes help to gain control over the electromagnetic fields. Recent studies have put stress on backward and forward scattering control $[23,26]$, near-field to far-field control, and possibilities opened for antennas [24]. Such a control over the scattered field requires the existence of two overlapping modes, which excludes single electric dipole coils or magnetic dipole coils [Fig. 2(b)]. These works follow a path opened by the seminal paper published by Kerker et al. [22]. Although most of these studies have considered dielectric resonators, we show here that we can obtain the same effect using HMA. In the case of dielectric resonators, specific 
(a)

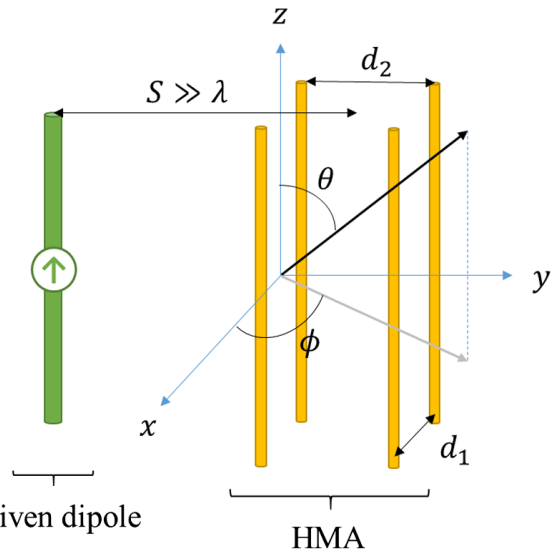

(b)

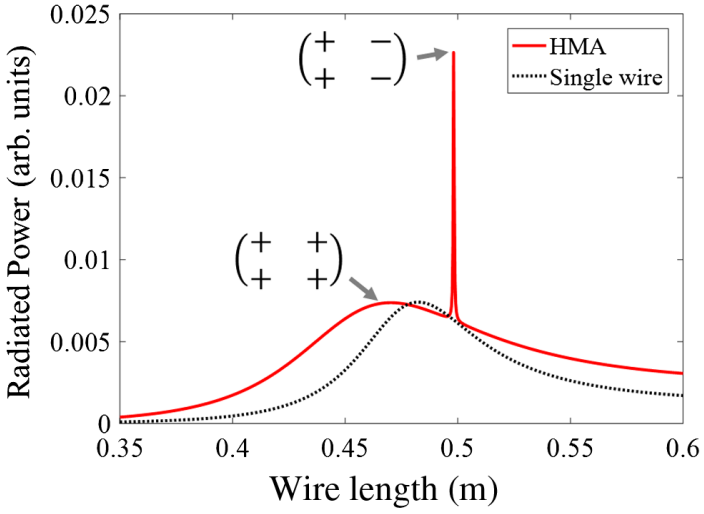

(c)

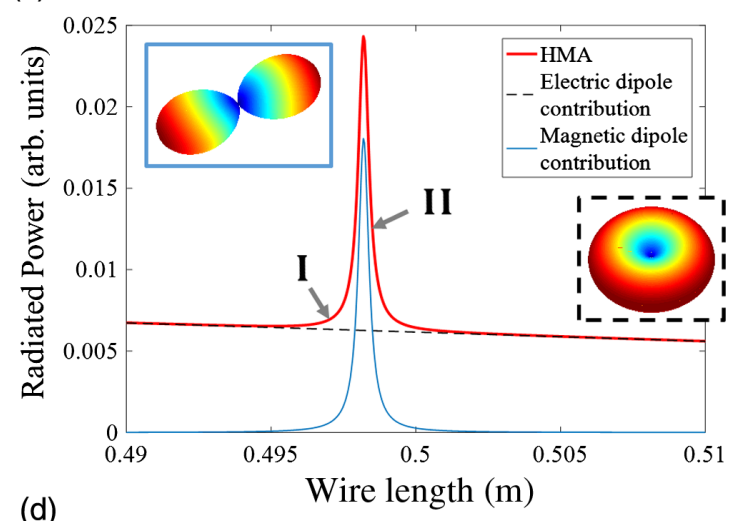

I II

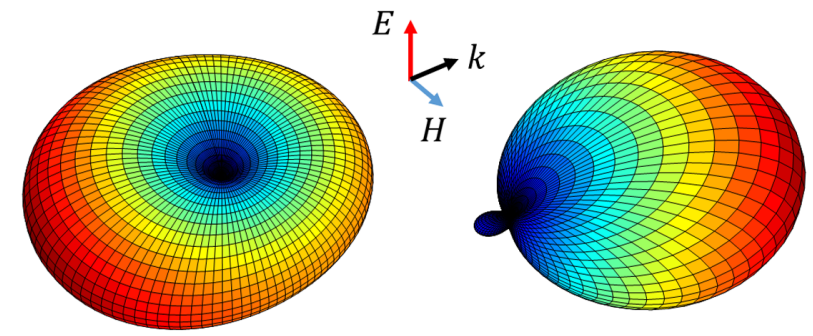

FIG. 2. (a) Sketch of the HMA configuration. Four metallic wires oriented along $z$ are placed at the four corners of a rectangle of sides $d_{1}=1.5 \mathrm{~cm}$ and $d_{2}=2 \mathrm{~cm}$. A fifth wire is used as a source located at a distance $S$ large compared to the wavelength at $297.2 \mathrm{MHz}$. Such a configuration allows us to study the response of the HMA with a plane wave excitation. All wires have a diameter of 2 mm. (b) Radiated power from the HMA (solid red line) as a function of the wire length (excitation frequency $297.2 \mathrm{MHz}$ is kept constant) is compared with the power radiated by a single wire (dashed black line) of the same varying length. The inset shows the phase of the current within the four wires of the HMA. This is evidence of the hybridization mechanism leading to a broad dipolar electric resonance and a sharp magnetic dipolar resonance. (c) Radiated power from the HMA on a narrow window (solid red line). Dashed black line and solid thin blue line show the isolated contributions of the two resonances excited in the HMA. The two insets show the radiation pattern of the magnetic dipolar resonance and the electric dipolar resonance. (d) Far-field radiation pattern at the two Kerker conditions (HMA lengths denoted above). The first one presents a reduction of the forward scattered field while a cancellation of the backward scattered field is observed at the second position.

index of refraction and diameter are required to control the number of excited modes and their overlap [24]. The dimensions and the spacing of the wires in our HMA structure are carefully chosen to obtain analog behavior. In our theoretical framework, the intensity radiated in the forward and backward directions is obtained with $\phi=\pi / 2$ and $\theta= \pm \pi / 2$, respectively; they are derived from $\mathrm{Eq}(3)$ :

$$
U_{\text {fwd,bwd }}=\frac{\left|\sum_{p=2}^{N} Z_{1 p}^{-1} e^{ \pm j y_{p}}\right|^{2}}{8 \pi^{2} c \epsilon_{0} \tan ^{2}\left(k \frac{L}{2}\right)} .
$$

As shown in Fig. 2(c), we can separate the response of the HMA onto the two different resonant contributions within the length range where these two modes coexist. The insets in Fig. 2(c) show the two radiation patterns obtained when the contributions are isolated. The electric dipolar resonance resembles a donut as the current distribution is invariant by rotation around the $z$ axis. This is not the case for the magnetic dipolar resonance, which shows a variation on both angles. Strong scattering anisotropy is obtained when these two behaviors are combined for wire lengths of 49.6 and $49.8 \mathrm{~cm}$ [Fig. 2(d)], due to interference between the two overlapping resonances. These specific lengths correspond to the Kerker conditions: a reduction of the forward scattering $(49.6 \mathrm{~cm})$ and a cancellation of the backward scattering $(49.8 \mathrm{~cm})$. They are obtained for precise interaction of electric and magnetic responses within the HMA. A detailed derivation of the conditions based on the existence of electric and magnetic dipolar modes in dielectric particles can be found in Ref. [26]. This is the first demonstration of scattering anisotropy or Kerker conditions from a single HMA. The following demonstrates that these Kerker conditions can be used to control the rf field distribution in a birdcage MRI volume coil. 


\section{B. Control of rf field within the MRI volume coil}

A sketch of the considered geometry is presented in Fig. 3(a). Our birdcage model is composed of $16 z$-oriented wires (called legs) of length $28 \mathrm{~cm}$ equally spaced on a cylinder of radius $13 \mathrm{~cm}$. The HMA is located at a distance $S=2.5 \mathrm{~cm}$ of the rightmost birdcage leg. As explained in the Introduction, the magnetic spin of hydrogen is sensitive to the $B_{1}^{+}$component of the magnetic field. This polarization is obtained while working in the rotating frame at the Larmor frequency considered. In the following, we use the convention $B_{1}^{+}=1 / 2\left[B_{x}+i B_{y}\right]$. Birdcage coils have been mostly used for their ability to provide a highly homogeneous $B_{1}^{+}$distribution when driven in quadrature. The current on each leg $I_{i}$ is described as $I_{i}=$ $I_{0}\left[\cos \left(\theta_{i}\right)-i \sin \left(\theta_{i}\right)\right]$, with $\theta_{i}=\{[2 \pi(i-1)] / N\}$, and $N$ the total number of legs. Knowing the current within each leg, we are able to calculate the $B_{1}^{+}$distribution using the BiotSavart formulation [29]. Details of the derivation are presented in the Supplemental Material [28].
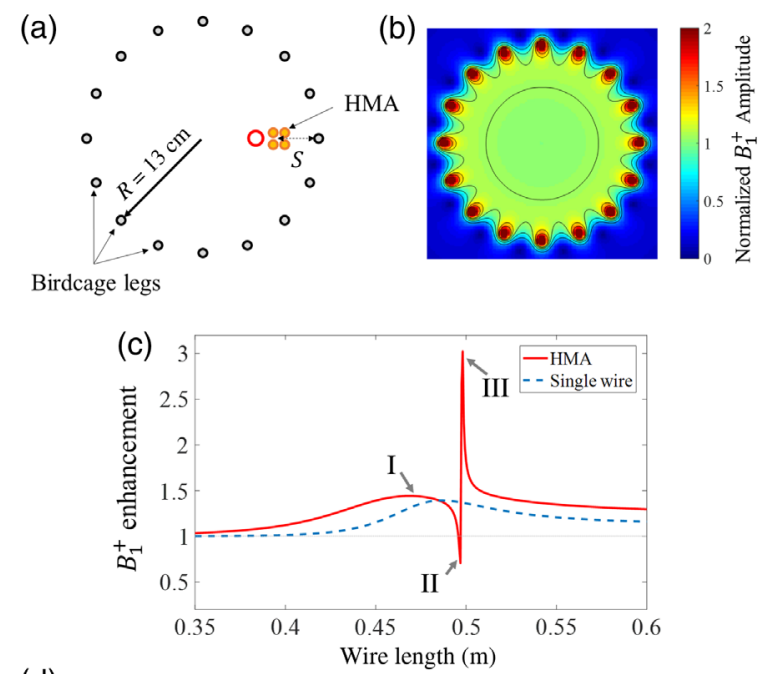

(d)
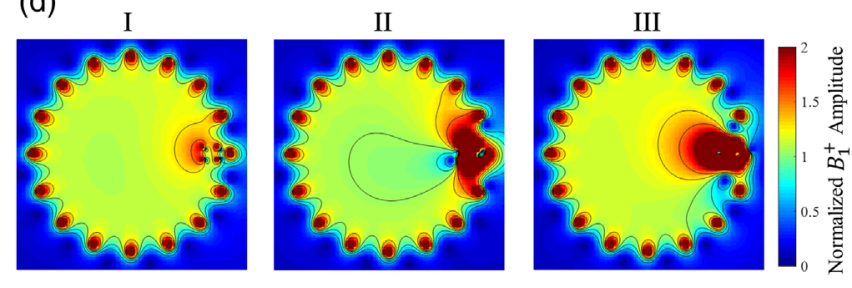

FIG. 3. (a) Sketch of the considered geometry for the insertion of the HMA inside a birdcage model. The birdcage is composed of 16 legs on a $26-\mathrm{cm}$-diameter cylinder. The legs are $28 \mathrm{~cm}$ long and the HMA is located at $S=2.5 \mathrm{~cm}$ from the first leg. Red circle denotes the ROI for the $B_{1}^{+}$enhancement plot. (b) $B_{1}^{+}$ amplitude calculated in a quadrature driven empty birdcage. (c) $B_{1}^{+}$enhancement in the ROI with respect to the empty birdcage in function of the HMA length. The solid red line corresponds to the HMA and the blue dashed line corresponds to the single wire case. (d) Calculated $B_{1}^{+}$amplitude maps with the HMA for three lengths of interest shown in (c), respectively, 47.5, 49.6 , and $49.8 \mathrm{~cm}$.

$$
B(x, y)=\left(\begin{array}{c}
B_{x} \\
B_{y}
\end{array}\right)=\frac{I \mu_{0}}{4 \pi\left(x^{2}+y^{2}\right) \sqrt{x^{2}+y^{2}+\frac{L^{2}}{4}}}\left(\begin{array}{c}
y L \\
-x L
\end{array}\right) .
$$

These equations give the in-plane components of the magnetic field obtained in the median plane of a $z$-oriented thin wire of length $L$ located in $(0,0)$. The Biot-Savart quasistatic approximation is valid with an empty birdcage coil as the diameter represents a quarter of the wavelength. The amplitude of $B_{1}^{+}$is shown in Fig. 3(b); it is used as a reference value. In order to obtain the currents on the HMA wires, we derive the impedance matrix when considering that the driven element is in the near field of the HMA $(S=2.5 \mathrm{~cm})$. Once we have the current distribution of the HMA, we can use Eq. (6) to calculate the magnetic field produced by the 16 birdcage legs and the four HMA wires with varying lengths. Then, a coherent sum of all the magnetic fields is realized before extracting the amplitude of $B_{1}^{+}$. The results for varying HMA lengths are presented in Figs. 3(c) and 3(d). The graph shows the $B_{1}^{+}$enhancement in the region of interest (ROI) delimited in the sketch (the red circle is $1 \mathrm{~cm}$ away from the HMA). The $B_{1}^{+}$ amplitude maps are given for three specific HMA lengths labeled on the solid red line. Once again we can observe a broad enhancement $(+50 \%)$ of the $B_{1}^{+}$field close to the HMA between 40 and $49 \mathrm{~cm}$. A sharp resonant effect is observed around $50 \mathrm{~cm}$ which shows a cancellation of the $B_{1}^{+}$amplitude at $49.6 \mathrm{~cm}$ followed by a threefold increase of the $B_{1}^{+}$amplitude close to the HMA at $49.8 \mathrm{~cm}$. These calculations demonstrate that the HMA scattering properties have a significant impact on the circularly polarized $B_{1}^{+}$ near-field amplitude in the birdcage coil. It is also very clear that the mode hybridization occurring in the HMA structure outperforms the single wire case in terms of bandwidth and enhancement factor. Moreover, the enhancement and the reduction of the $B_{1}^{+}$amplitude both have a valuable impact on the MRI acquisition: it offers the ability to measure areas of the head which did not receive enough power initially and can also reduce the amplitude in the overexposed area that could potentially present safety issues [30].

\section{Experimental demonstration of Kerker conditions in MRI}

MRI acquisition can be affected by a combination of many parameters, such as the size, the shape, and the relative permittivity of the subject. First, we decide to use a spherical phantom with low permittivity in order to strictly validate the theoretical behavior of the HMA. We realized a set of telescopic resonators made of four coupled metallic rods (radius equal to $0.5 \mathrm{~mm}$ ) located at the corners of a rectangle of sides $d_{1}=1.5 \mathrm{~cm}$ and $d_{2}=2 \mathrm{~cm}$. The length of the HMA can be tuned between 20 and $100 \mathrm{~cm}$. The telescopic configuration and the size of the HMA were 
(a)

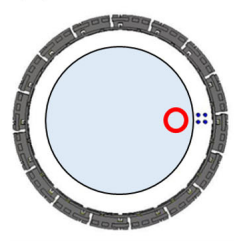

(b)

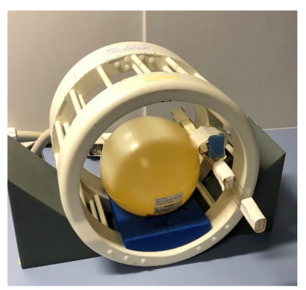

(c)

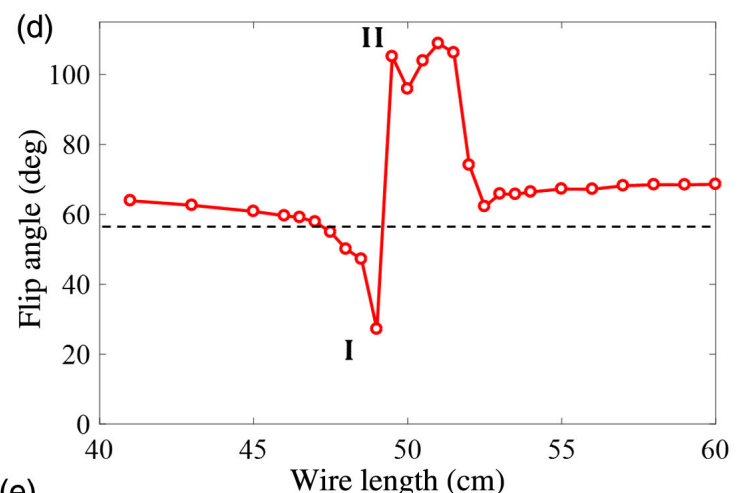

(e)
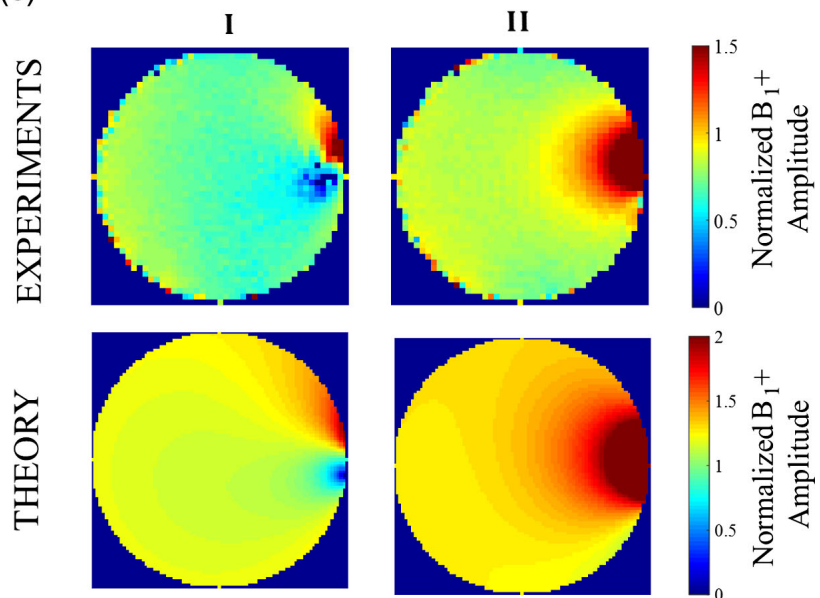

FIG. 4. (a) Schematic view of experimental configuration inside the 7 T MRI: birdcage coil (dark gray), HMA (4 blue rods), and oil phantom $\epsilon_{r}=3.4$ (light gray). (b),(c) Pictures of the experimental setup, positioning of the HMA in the birdcage coil and into the MRI scanner. (d) Average flip-angle magnitude in the ROI [red circle in (a)] as a function of the HMA length. The dashed line indicates the reference value (without HMA). (e) Top: Normalized axial $B_{1}^{+}$maps measured for two lengths of interest, namely, 49 and $49.5 \mathrm{~cm}$. Each corresponds to the Kerker conditions with an enhancement or cancellation of the $B_{1}^{+}$field close to the HMA. Bottom: Normalized field maps obtained from the theoretical calculations for the two Kerker conditions. HMA length from left to right at the calculated Kerker conditions (49.6 and $49.8 \mathrm{~cm}$, respectively). A circular mask is applied to reproduce the phantom dimensions.

selected to keep the same configuration in all the experiments (i.e., to have continuously varying configurations). Experiments were performed using a birdcage head coil 1Tx/1Rx (Invivo Corp., Gainesville, FL) and a 16-cmdiameter spherical phantom filled with oil [Fig. 4(b)] on a 7 T Magnetom MRI (Siemens Healthineers, Erlangen, Germany) [Fig. 4(c)]. A sketch of the experimental configuration is given in Fig. 4(a). $B_{1}^{+}$maps were acquired with a magnetization-prepared turbo-Fast Low Angle Shot sequence (XFL) [31] with varying HMA lengths. In order to quantify the effect of the HMA, we have defined a ROI depicted by a red circle in Fig. 4(a). The images were acquired for 30 different lengths of the HMA (all images are shown in Supplemental Material [28]) and we have computed the averaged flip-angle values (proportional to $B_{1}^{+}$) in the ROI for each length. Figure 4(d) shows the obtained values of averaged flip angle with respect to the length, while the dashed line shows the averaged value in the same region without HMA. When the HMA is present we observe an increase of $B_{1}^{+}$field in the phantom on the right, i.e., near the HMA. This effect is related to the increase of the local magnetic field when a resonant mode is excited. When the length is further tuned, we are able to decrease drastically the $B_{1}^{+}$field when the backward scattering condition is reached at a length equal to $49 \mathrm{~cm}$. Then we observe an even stronger increase in the near $B_{1}^{+}$field at the forward scattering condition length $(49.5 \mathrm{~cm})$. The experimental results are compared to theoretical prediction and show a very good agreement which confirms our interpretation of the HMA behavior.

\section{D. $r f$ inhomogeneities reduction in realistic phantoms}

In this experimental section, we demonstrate that the HMA can reduce the rf field inhomogeneities when inserted close to a phantom reproducing the electromagnetic behavior of the human body. We use a 16-cm-diameter sphere filled with an agar-agar gel with high permittivity $\epsilon_{r}=74.2$ and high conductivity $\sigma=0.87 \mathrm{~S} / \mathrm{m}$. The $B_{1}^{+}$distribution observed is highly inhomogeneous with a maximum at the center surrounded by a ringlike minimum region when measured with the birdcage alone [Fig. 5(b)]. This illustrates 
the impact of the wavelength reduction in the human body, as previously described in the Introduction (Fig. 1). A sketch of the experimental configurations is given in Fig. 5(a). The HMA is located on the right-hand side of the phantom, and three ROIs are highlighted. The black circle corresponds to the whole phantom while the green and red circles are located in the center and in the vicinity of the HMA, respectively. $B_{1}^{+}$ maps are measured with varying HMA lengths between 30 and $60 \mathrm{~cm}$ with the same telescopic metallic wires. Figure 5(c) presents the $B_{1}^{+}$maps obtained for two lengths of interest corresponding to the maximum (at $46.5 \mathrm{~cm}$ ) and minimum (at $49.5 \mathrm{~cm}$ ) $B_{1}^{+}$amplitude in the vicinity of the HMA. The resonances within the HMA structure are responsible for the enhancement and the cancellation of the local $B_{1}^{+}$amplitude. More importantly than the local

(a)

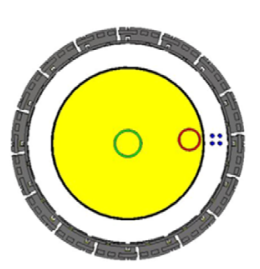

(b)
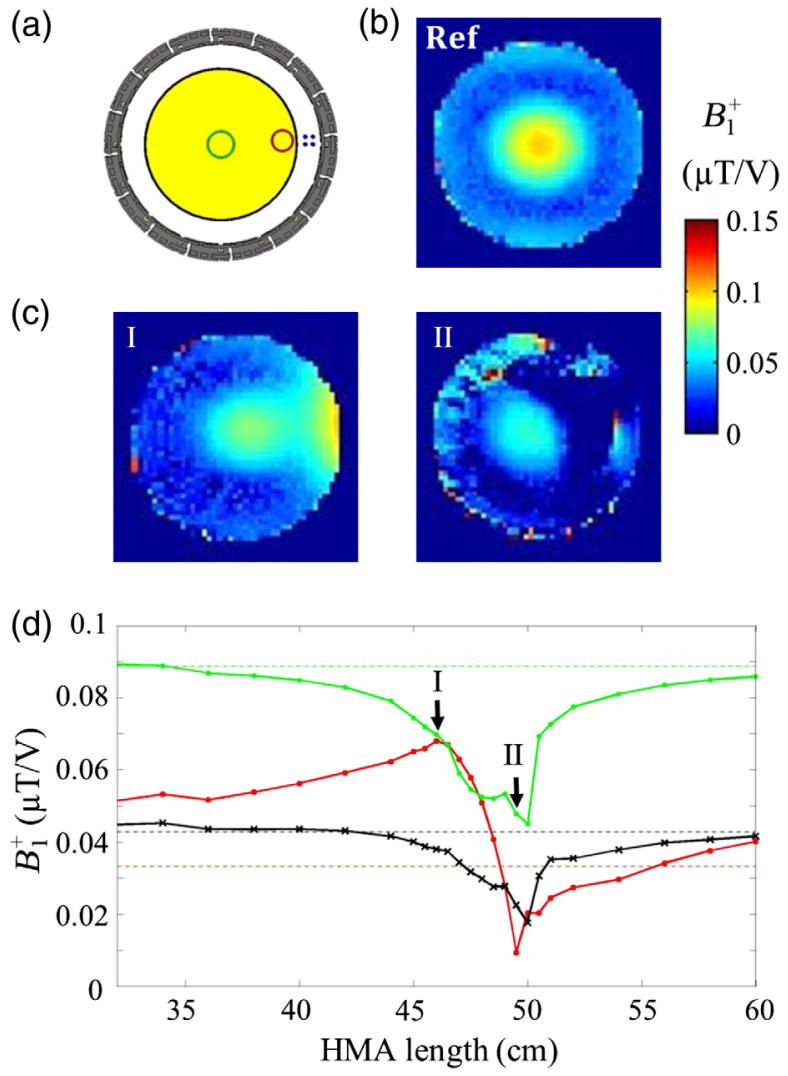

FIG. 5. (a) Schematic view of experimental configuration inside the 7 T MRI: birdcage coil (dark gray), HMA (4 blue rods), and phantom (yellow). (b),(c) Measured $B_{1}^{+}$maps (in $\mu \mathrm{T} / \mathrm{V})$ of the gel spherical phantom $\left(\epsilon_{r}=74.2, \sigma=0.87 \mathrm{~S} / \mathrm{m}\right)$ (b) without HMA and (c) for various HMA lengths: (I) $46.5 \mathrm{~cm}$ and (II) $49.5 \mathrm{~cm}$. (d) Average $B_{1}^{+}$magnitude in the three colormarked ROIs (a) as a function of the HMA length. The black solid line corresponds to the whole phantom. The green solid line corresponds to the central region of the phantom ( $1.85 \mathrm{~cm}$ radius). The red solid line corresponds to the right-hand region of the phantom ( $1.5 \mathrm{~cm}$ radius). The horizontal dotted lines indicate the reference value (without HMA) for each ROI. The three vertical arrows denote the three lengths of interest depicted above. enhancement, the presence of the HMA also affects the amplitude of the central spot which is highly beneficial to the homogeneity of the rf field. This effect is clearly perceived in Fig. 5(d), representing the field average in the three ROIs depicted in Fig. 5(a). Indeed, one can see that the values averaged in the central spot (green line) and in the vicinity of the HMA (red line) are equal for a HMA length of $46.5 \mathrm{~cm}$. Moreover, Fig. 5(c) shows that the $B_{1}^{+}$amplitude is homogeneous in the area between the HMA and the center of the sphere, contrary to the strong dispersion of values obtained with the birdcage alone denoted by the dashed line in Fig. 5(d). Note that this control over the rf field distribution does not perturb the matching of the birdcage coil. The measured total transmitted power at a constant input voltage $(133 \mathrm{~V})$ decreases by $2 \%$ at $46.5 \mathrm{~cm}$ length, whereas it reaches a minimum of $6 \%$ at $49.5 \mathrm{~cm}$ length. This result clearly demonstrates our ability to reduce the dispersion of the rf field amplitude in the phantom without inducing perturbations over the original coil properties.

Finally, we perform a comparison of the performances obtained on a specific anthropomorphic mannequin (SAM) phantom (SPEAG, Zurich, Switzerland) with our HMA and the reference high dielectric constant pads used in literature [32] based on a mixture of $\mathrm{BaTiO}_{3}$ and water. $B_{1}^{+}$maps are acquired with an actual flip-angle imaging sequence [33]. The results for the birdcage with SAM alone are presented in Fig. 6(a). Once again, the characteristic inhomogeneous rf field pattern is obtained. The permittivity of the $\mathrm{BaTiO}_{3}$ mixture designed is close to $\epsilon_{r}=220$, which is optimal for a 1-cm-thick pad [5]. The mixture is sealed in a plastic box of dimensions $12 \times 10 \times 1 \mathrm{~cm}^{3}$ and placed on the righthand side of the SAM phantom. The effect of the $\mathrm{BaTiO}_{3}$ pad is presented in Fig. 6(b). A strong amplitude increase is observed in the area close to the pad. However, the pad configuration is not able to fill the gap between the side of the phantom and the central spot, meaning that the dispersion of $B_{1}^{+}$amplitude values remains broad. A final measurement is realized with a $42-\mathrm{cm}$-long HMA placed at $2 \mathrm{~cm}$ from the right-hand side of the phantom. The HMA provides a similar enhancement of the $B_{1}^{+}$amplitude on the right-hand side of the SAM phantom. Nonetheless, the HMA configuration is such that we are able to merge the two high-amplitude spots into one large area with low dispersion in terms of $B_{1}^{+}$amplitude values. This is highly beneficial to the SNR and biological contrast recovery for in vivo MRI applications. Furthermore, it clearly shows the benefits of the metamaterial approach developed here compared to the actual benchmark in terms of $\mathrm{rf}$ passive shimming.

\section{E. Signal-to-noise ratio evaluation}

In order to fully assess the performances of our rf shimming approach, we explore the effect of the HMA structure in terms of signal-to-noise ratio. In this section, we compare the signal-to-noise ratio obtained with the 
(a)

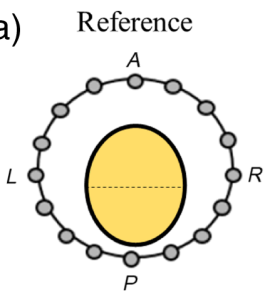

(b) $\quad \mathrm{BaTiO}_{3} \mathrm{Pad}$
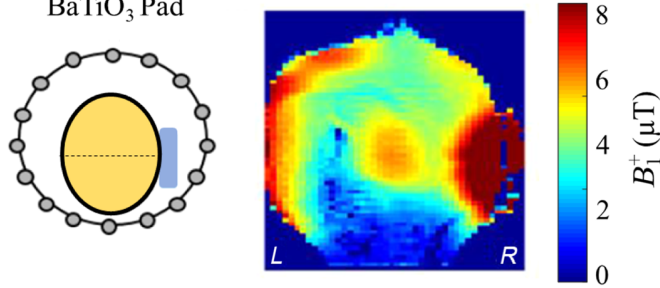

(c) $\mathrm{HMA}-42 \mathrm{~cm}$
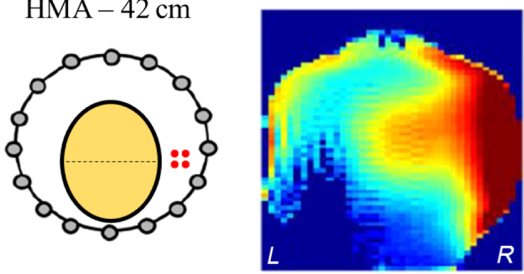

FIG. 6. (a)-(c) Left: Schematic view of the experimental configurations inside the 7 T MRI: birdcage legs in gray circles, specific anthropomorphic mannequin (SAM) in yellow, positioning of the $\mathrm{BaTiO}_{3}$ pad in blue (b) and HMA in red dots (c). The horizontal dotted line shows the imaging plane. Right: Measured $B_{1}^{+}$coronal maps (in $\mu \mathrm{T}$ ) of the SAM phantom: (a) with SAM only, (b) in the presence of a $\mathrm{BaTiO}_{3}$ pad on the right-hand side, and (c) replacing the pad with a 42-cm-long HMA. HMA is located $2 \mathrm{~cm}$ away from the phantom.

birdcage alone as a reference and observe the impact of the HMA when inserted within the coil. To do so, we use a gradient echo sequence to obtain a proton weighted image of the phantom. These results are presented in Figs. 7(a) and 7(b) for the reference case and in the presence of HMA. In a second measurement, we use XFL sequence to acquire the excitation profile. These two maps are then used to calculate the SNR maps independently from the flip-angle heterogeneities [Figs. 7(c) and 7(d)]. Finally, we compute the SNR enhancement map [Fig. 7(e)] as the ratio of the two corrected SNRs measured with and without the HMA. More details are presented in Sec. IV. All the measurements are done with the same input power and sequence parameters. First of all, there is almost no decay in the coil efficiency once the HMA is inserted. The autocalibration of the system leads only to a $1 \%$ increase once the HMA is set in position. Figures 7(a) and 7(b) demonstrate a local enhancement of the signal in the vicinity of the HMA. This is a consequence of the $\mathrm{rf}$ shimming observed in the previous results. Nevertheless, Figs. 7(c) and 7(d) show that even at the same input power, and after correction for flip-angle heterogeneity bias, receiving SNR is locally improved by the HMA insertion. This result can be (a)

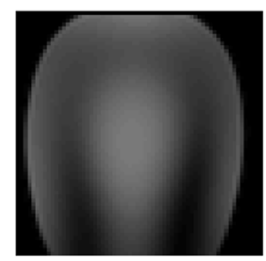

(b)

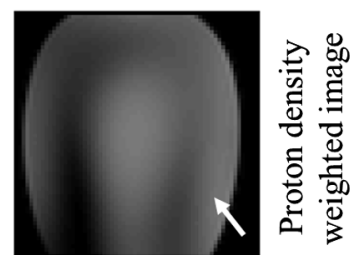

(c)

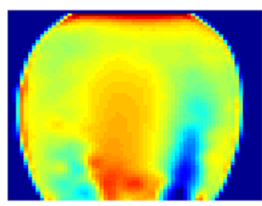

(d)

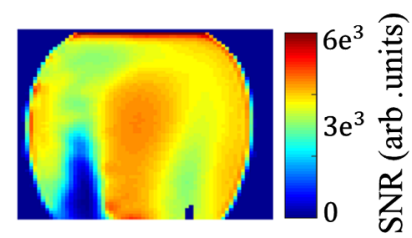

(e)

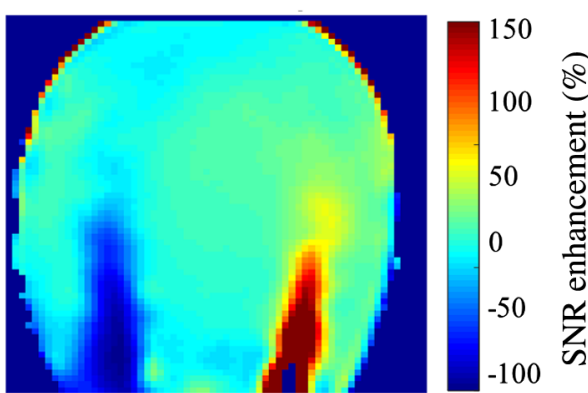

FIG. 7. SNR measurement with and without HMA on SAM phantom. (a) Proton density weighted (PDW) coronal image with birdcage and (b) with 42-cm-long HMA inserted (metamaterial along the right-hand side of the phantom). Local enhancement can be visually identified by the white arrow along the HMA. (c) Corrected SNR maps (arbitrary units) obtained with the birdcage alone and (d) in presence of HMA. (e) Calculated SNR enhancement map in percentage. It confirms a strong SNR enhancement close to the HMA but also a slight improvement on a relatively large part of the phantom volume.

interpreted through the reciprocity principle [34], where gain in transmission can also be observed in reception from the linearly polarized magnetic field emitted by the HMA. Moreover, it also goes with a better noise figure in reception as the noise level measured at the analog-to-digital converter is reduced by $9 \%$. Consequently, we obtain an enhancement of the SNR over the right hemisphere of the phantom. We observe a maximum of $150 \%$ enhancement in the original dark spot produced by the birdcage alone. One can notice a reduction of the SNR within the dark spot located on the opposite side, as previously observed with a single dielectric pad. This results show that besides the clear advantage of local $B_{1}^{+}$enhancement provided by the HMA, the reception path benefits also from the presence of the HMA within the birdcage coil.

\section{CONCLUSION}

In conclusion, the results we present here demonstrate a strong impact of HMA on the $B_{1}^{+}$field and local SNR for UHF MRIs. Besides their clear effect on the rf field distribution, HMA structures have the potential to overcome 
limitations of the dielectric pads. As metallic metamaterials are not subject to aging, they will keep their performance along time; moreover, they are cost effective and very easy to manufacture. The HMA equivalent dielectric constant is also tunable by simply modifying the length and the distance between the rods. Thus, in a first approach, HMAs could be used similarly to dielectric pads but allowing a higher degree of control in the rf field distribution. In addition, we show that we could strongly increase or reduce the $B_{1}^{+}$field when the Kerker scattering conditions are set. Decreasing the $B_{1}^{+}$ field in specific regions may also have applications for shielding metallic implants [30], for example. The developed solution could be directly used to improve functional MRI imaging. Indeed, it has not been fully optimized for imaging but should be seen as a proof of concept of the control of the rf emission for UHF MRI. Future work will consider a combination of several HMA structures inside the coil to not only restore locally the $B_{1}^{+}$excitation but also improve the $B_{1}^{+}$dispersion in the whole brain at $7 \mathrm{~T}$ [35]. In addition to the padding route, a different approach could be developed considering the HMAs and the birdcage coil as a whole, which should be designed in order to be insensitive to individual variability [36].

\section{METHOD}

Validation experiments were performed on a homemade 16-cm-diameter spherical phantom containing $1 \%$ agar and $0.4 \% \mathrm{NaCl}\left(\epsilon_{r}=74.5, \sigma=0.78 \mathrm{~S} / \mathrm{m}\right)$ and on a specific anthropomorphic mannequin phantom (SPEAG, Zurich, Switzerland) filled with HT0300 liquid $\left(\epsilon_{r}=45.3\right.$, $\sigma=0.87 \mathrm{~S} / \mathrm{m}$ ) using a birdcage $1 \mathrm{Tx} / 1 \mathrm{Rx}$ head coil (Invivo Corp., Gainesville, FL) in a 7 T Magnetom MRI scanner (Siemens Healthineers, Erlangen, Germany).

Experimental $B_{1}^{+}$maps were acquired with the XFL MRI sequence $[31,37,38]$ in the coronal direction. It is based on a 2D Turbo-FLASH readout sequence, acquired once with a $5 \mathrm{~ms} 90^{\circ}$ Shinnar-Leroux minimum-phase saturation pulse (SAT) [37,39] with a time-to-bandwidth product of 9, and once without (REF). Sequence parameters for a $4 \mathrm{~mm}$ in-plane resolution with a field of view of $256 \times 256 \times$ $176 \mathrm{~mm}^{3}$ were $\mathrm{TR}=20 \mathrm{~s}, \mathrm{TE}=3.06 \mathrm{~ms}, \mathrm{FA}=7^{\circ}$, $\mathrm{BW}=1560 \mathrm{~Hz} / \mathrm{px}$. The XFL acquisition time lasted $40 \mathrm{~s}$ without any acceleration factor. Considering that longitudinal relaxation can be neglected between the SAT pulse and the first REF pulse, a flip-angle map can be calculated with the following expression:

$$
\alpha=\cos ^{-1}\left(\frac{S_{\mathrm{SAT}}}{S_{\mathrm{REF}}}\right)
$$

where $S_{\mathrm{SAT}}$ and $S_{\mathrm{REF}}$ are saturation and reference magnitude signal images. The $B_{1}^{+}$field map can then be extracted using the following formula:

$$
B_{1}^{+}=\frac{\alpha}{360 \gamma \int_{0}^{\tau} \operatorname{SAT}(t) d t}
$$

where $\alpha$ is the measured flip angle in degrees, $\gamma$ is the gyromagnetic ratio in $\mathrm{Hz} / \mu \mathrm{T}, \tau$ is the saturation pulse duration, and $\operatorname{SAT}(t)$ is the waveform of the saturation pulse in volts.

Proton density weighted images used in Sec. IIE are obtained with a gradient echo sequence with $\mathrm{TR}=5 \mathrm{~s}$, $\mathrm{TE}=1.9 \mathrm{~ms}, \mathrm{FA}=90^{\circ}, \mathrm{BW}=1563 \mathrm{~Hz} / \mathrm{px}$, field of view of $256 \mathrm{~mm}^{2}$ on $64 \times 64$ pixels with $4 \mathrm{~mm}$ isotropic voxel size. For this proton density weighting with $\mathrm{TR}>5 T_{1}$, the signal $s_{\mathrm{GRE}}$ obtained in the function of the position $r$ is

$$
s_{\mathrm{GRE}}(r)=M_{0}(r) E_{2} R(r) \sin \theta(r),
$$

with $M_{0}(r)$ the longitudinal magnetization available, $R(r)$ the reception profile, the flip-angle map $\theta(r)$, and the factor $E_{2}=e^{-\mathrm{TE} / T_{2}^{*}}$. This last factor has a reduced impact since TE is lower than $T_{2}^{*}$. The excitation profile $\theta(r)$ is removed from the signal expression in order to compute the corrected SNR map as follows:

$$
\operatorname{SNR}(r)=\frac{\left\langle M_{0}(r) R(r)\right\rangle}{\operatorname{std}\left(s_{\mathrm{GRE}-0 \mathrm{~V}}\right)},
$$

where $s_{\mathrm{GRE}-0 \mathrm{~V}}$ is a measure of real noise with no $\mathrm{rf}$ power during the sequence.

\section{ACKNOWLEDGMENTS}

The project leading to this publication has received funding from Excellence Initiative of Aix-Marseille UniversityA*MIDEX, a French "Investissements d'Avenir" programme. It has been partly funded by France Life Imaging Grant No. ANR-11-INBS-0006. It has received funding from the European Union's Horizon 2020 Research and Innovation programme under Grant Agreement No. 736937. We also thank Institut Carnot STAR for support.

R. A., M. D., and S.E. worked on the physics and the basic principles of the HMA for UHF-MRI. M. D., R. A., L. L., A. V., and S.E. worked on the modeling and the design of the HMA. MRI experiments were conducted by L. L., Z. R., and A. V. A. A. and F. M. developed the $B_{1}^{+}$ mapping MRI sequence. All authors contributed to the analysis and redaction of the manuscript.

M. D. and L. L. contributed equally to this work.

[1] R. Pohmann, O. Speck, and K. Scheffler, Signal-to-Noise Ratio and MR Tissue Parameters in Human Brain Imaging at 3, 7, and 9.4 Tesla Using Current Receive Coil Arrays, Magn. Reson. Med. 75, 801 (2016).

[2] C. Boutet, M. Chupin, S. Lehéricy, L. Marrakchi-Kacem, S. Epelbaum, C. Poupon, C. Wiggins, A. Vignaud, D. Hasboun, B. Defontaines et al., Detection of Volume Loss in 
Hippocampal Layers in Alzheimer's Disease Using 7 T MRI: A Feasibility Study, Neuroimage Clin. 5, 341 (2014).

[3] P.-F. Van de Moortele, C. Akgun, G. Adriany, S. Moeller, J. Ritter, C. M. Collins, M. B. Smith, J. T. Vaughan, K. Uğurbil, B1 Destructive Interferences and Spatial Phase Patterns at 7 T with a Head Transceiver Array Coil, Magn. Reson. Med. 54, 1503 (2005).

[4] A. G. Webb, Dielectric Materials in Magnetic Resonance, Concepts Magn. Reson., Part A 38A, 148 (2011).

[5] A. L. Neves, L. Leroi, Z. Raolison, N. Cochinaire, T. Letertre, R. Abdeddaim, S. Enoch, J. Wenger, J. Berthelot, A.-L. Adenot-Engelvin et al., Compressed Perovskite Aqueous Mixtures Near Their Phase Transitions Show Very High Permittivities: New Prospects for High-Field MRI Dielectric Shimming, Magn. Reson. Med. 79, 1753 (2018).

[6] T. P. A. O'Reilly, A. G. Webb, and W. M. Brink, Practical Improvements in the Design of High Permittivity Pads for Dielectric Shimming in Neuroimaging at 7 T, J. Magn. Reson. 270, 108 (2016).

[7] W. Grissom, C.-y. Yip, Z. Zhang, V. A. Stenger, J. A. Fessler, and D. C. Noll, Spatial Domain Method for the Design of RF Pulses in Multicoil Parallel Excitation, Magn. Reson. Med. 56, 620 (2006).

[8] FDA 510(k) premarket approval under No. K170840 for Magnetom Terra 7 T, Oct. 2017.

[9] M. C. K. Wiltshire, J. B. Pendry, I. R. Young, D. J. Larkman, D. J. Gilderdale, and J. V. Hajnal, Microstructured Magnetic Materials for RF Flux Guides in Magnetic Resonance Imaging, Science 291, 849 (2001).

[10] M. J. Freire, L. Jelinek, R. Marques, and M. Lapine, On the Applications of $\mu=-1$ Metamaterial Lenses for Magnetic Resonance Imaging, J. Magn. Reson. 203, 81 (2010).

[11] X. Radu, D. Garray, and C. Craeye, Toward a Wire Medium Endoscope for MRI Imaging, Metamaterials 3, 90 (2009).

[12] C. L. Holloway, E. F. Kuester, J. A. Gordon, J. O'Hara, J. Booth, and D. R. Smith, An Overview of the Theory and Applications of Metasurfaces: The Two-Dimensional Equivalents of Metamaterials, IEEE Trans. Antennas Propag. 54, 10 (2012).

[13] A. P. Slobozhanyuk, A. N. Poddubny, A. J. E. Raaijmakers, C. A. T. van den Berg, A. V. Kozachenko, I. A. Dubrovina, I. V. Melchakova, Y. S. Kivshar, and P. A. Belov, Enhancement of Magnetic Resonance Imaging with Metasurfaces, Adv. Mater. 28, 1832 (2016).

[14] A. V. Shchelokova, C. A. T. van den Berg, D. A. Dobrykh, S. B. Glybovski, M. A. Zubkov, E. A. Brui, D. S. Dmitriev, A. V. Kozachenko, A. Y. Efimtcev, A. V. Sokolov et al., Volumetric Wireless Coil Based on Periodically Coupled Split-Loop Resonators for Clinical Wrist Imaging, Magn. Reson. Med. 80, 1726 (2018).

[15] A. V. Shchelokova, A. P. Slobozhanyuk, I. V. Melchakova, S. B. Glybovski, A. G. Webb, Y. S. Kivshar, and P. A. Belov, Locally Enhanced Image Quality with Tunable Hybrid Metasurfaces, Phys. Rev. Applied 9, 014020 (2018).

[16] A. V. Shchelokova, A. P. Slobozhanyuk, P. de Bruin, I. Zivkovic, E. Kallos, P. A. Belov, and A. Webb, Experimental Investigation of a Metasurface Resonator for In Vivo Imaging at 1.5 T, J. Magn. Reson. 286, 78 (2018).
[17] C. Jouvaud, R. Abdeddaim, B. Larrat, and J. De Rosny, Volume Coil Based on Hybridized Resonators for Magnetic Resonance Imaging, Appl. Phys. Lett. 108, 023503 (2016).

[18] I. R. O. Connell, K. M. Gilbert, M. A. Abou-Khousa, and R. S. Menon, Design of a Parallel Transmit Head Coil at 7 T with Magnetic Wall Distributed Filters, IEEE Trans. Med. Imaging 34, 836 (2015).

[19] A. A. Hurshkainen, T. A. Derzhavskaya, S. B. Glybovski, I. J. Voogt, I. V. Melchakova, C. A. T. van den Berg, and A. J. E. Raaijmakers, Element Decoupling of $7 T$ Dipole Body Arrays by EBG Metasurface Structures: Experimental Verification, J. Magn. Reson. 269, 87 (2016).

[20] E. Georget, M. Luong, A. Vignaud, E. Giacomini, E. Chazel, G. Ferrand, A. Amadon, F. Mauconduit, S. Enoch, G. Tayeb et al., Stacked Magnetic Resonators for MRI RF Coils Decoupling, J. Magn. Reson. 275, 11 (2017).

[21] R. Schmidt, A. Slobozhanyuk, P. Belov, and A. Webb, Flexible and Compact Hybrid Metasurfaces for Enhanced Ultra High Field In Vivo Magnetic Resonance Imaging, Sci. Rep. 7, 1678 (2017).

[22] M. Kerker, D.-S. Wang, and C. L. Giles, Electromagnetic Scattering by Magnetic Spheres, J. Opt. Soc. Am. 73, 765 (1983).

[23] J.-M. Geffrin, B. García-Cámara, R. Gómez-Medina, P. Albella, L. S. Froufe-Pérez, C. Eyraud, A. Litman, R. Vaillon, F. González, M. Nieto-Vesperinas et al., Magnetic and Electric Coherence in Forward- and BackScattered Electromagnetic Waves by a Single Dielectric Subwavelength Sphere, Nat. Commun. 3, 1171 (2012).

[24] B. Rolly, J.-M. Geffrin, R. Abdeddaim, B. Stout, and N. Bonod, Controllable Emission of a Dipolar Source Coupled with a Magneto-Dielectric Resonant Subwavelength Scatterer, Sci. Rep. 3, 3063 (2013).

[25] N. Meinzer, W. L. Barnes, and I. R. Hooper, Plasmonic Meta-Atoms and Metasurfaces, Nat. Photonics 8, 889 (2014).

[26] M. Nieto-Vesperinas, R. Gomez-Medina, and J. J. Saenz, Angle-Suppressed Scattering and Optical Forces on Submicrometer Dielectric Particles, J. Opt. Soc. Am. A 28, 54 (2011).

[27] S. J. Orfanidis, Electromagnetic Waves and Antennas (Rutgers University, New Brunswick, NJ, 2002).

[28] See Supplemental Material at http://link.aps.org/ supplemental/10.1103/PhysRevX.8.031083 for details (1) experimental flip angle maps in function of HMA length, (2) details on impedance matrix derivation, and (3) details on Biot-Savart derivation and $B_{1}^{+}$field.

[29] P. Boissoles, Mathematical and Numerical Problems from Nuclear Magnetic Resonance Imaging, Ph.D. thesis, Université Rennes 1, 2005.

[30] M. J. P. van Osch and A. G. Webb, Safety of Ultra-High Field MRI: What Are the Specific Risks?, Curr. Radiol. Rep. 2, 61 (2014).

[31] A. Amadon, F. Mauconduit, A. Vignaud, and N. Boulant, Slice Profile Corrections in theXFLl (MagnetizationPrepared Turbo-Flash) B1-Mapping Sequence, in Proceedings of the 20th Annual Meeting of ISMRM, Toronto, 2015, Vol. 23, p. 2377. 
[32] W. M. Teeuwisse, W. M. Brink, K. N. Haines, and A. G. Webb, Simulations of High Permittivity Materials for 7 T Neuroimaging and Evaluation of a New Barium TitanateBased Dielectric, Magn. Reson. Med. 67, 912 (2012).

[33] V. L. Yarnykh, Actual Flip-Angle Imaging in the Pulsed Steady State: A Method for Rapid Three-Dimensional Mapping of the Transmitted Radiofrequency Field, Magn. Reson. Med. 57, 192 (2007).

[34] D. Hoult, The Principle of Reciprocity in Signal Strength Calculations -A Mathematical Guide, Concepts Magn. Reson., Part A 12, 173 (2000).

[35] R. Abdeddaim, S. Enoch, P. Sabouroux, G. Tayeb, N. Bonod, A. Vignaud, B. Larrat, E. Georget, and L. Leroi, Method for Controlling the Distribution of the RF Magnetic Field in a Magnetic Resonance Imaging System, France Patent No. WO/2017/198914 A1 (2017).
[36] V. Gras, A. Vignaud, A. Amadon, D. Bihan, and N. Boulant, Universal Pulses: A New Concept for Calibration-Free Parallel Transmission, Magn. Reson. Med. 77, 635 (2017).

[37] A. Amadon, N. Boulant, M. A. Cloos, E. Giacomini, C. J. Wiggins, M. Luong, G. Ferrand, and H. P. Fautz, B1-Mapping of an 8-Channel TX-Array over a HumanHead-Like Volume in Less than 2 Minutes: The XEP Sequence, in Proceedings of the 20th Annual Meeting of ISMRM, Stockholm, 2010, Vol. 18, p. 2828.

[38] S. Chung, D. Kim, E. Breton, and L. Axel, Rapid $B_{1}^{+}$ Mapping Using a Preconditioning RF Pulse with Turboflash Readout, Magn. Reson. Med. 64, 439 (2010).

[39] J. Pauly, P. Le Roux, D. Nishimura, and A. Macovski, Parameter Relations for the Shinnar-Le Roux Selective Excitation Pulse Design Algorithm (NMR Imaging), IEEE Trans. Med. Imaging 10, 53 (1991). 